\title{
38
}

\section{Co-ordination Functions in a SME Network}

\author{
Flavio Bonfatti and Paola Daniela Monari \\ University of Modena, Faculty of Engineering \\ clo CICAIA, via Campi 213/B, 41100 - Modena (Italy) \\ tel+3959 378514; fax+3959378515; e-mail bonfatti @ unimo.it
}

\author{
Paolo Paganelli \\ Democenter s.cons.r.l. \\ Viale Virgilio 55, 41100 - Modena (Italy); tel + 3959 848810; \\ fax+3959848630; e-mail democenter@mo.nettuno.it
}

\begin{abstract}
Construction of small-medium enterprise networks is a promising perspective in the industrial regions where such lean organisations prevail. SMEs present positive qualities to play the role of network nodes, but a critical problem is that of network production planning, since the nodes want to take part in the manufacturing process on a footing of equality. A solution to this problem in terms of planning policy formalization and supporting software tools is studied within the ESPRIT project 20723 - PLENT (PLanning small-medium Enterprise NeTworks). It is the aim of this paper to analyse and discuss the most important network coordination functions concerned with workload assignment to nodes.
\end{abstract}

Keywords

Enterprise network, Process modelling, Workload assignment.

\section{INTRODUCTION}

Much attention is presently paid to the idea of virtual enterprise, intended as a network of autonomous units that co-operate in satisfying market demand $[5,6,7,8]$. We can recall the large scale projects Manufacturing 21st (Japan), Agile Manufacturing (United States) and HMS - Holonic Manufacturing Systems, as well as the many national and international researches funded in the last few years, for instance within the ESPRIT programme. Unit leanness and specialisation, agile network configurability, responsibility subdivision, system co-ordination are key aspects of this promising organisational paradigm. 
While a great effort is spent in studying how to decompose large companies into specialised entities, and how to teach them flexibility and autonomy, we often disregard the huge number of small-medium enterprises (SMEs) that already present these qualities and could obtain significant benefits from participating in manufacturing networks. In particular: (i) more complex and technologically advanced products can be realised by putting together skills and design capabilities of the network nodes; (ii) higher production volumes are obtained by cumulating node capacities; (iii) fluctuations of market demand volumes are better borne by sharing workload peaks and shortages among the network nodes; (iv) the limited resources of each SME are better spent in technological innovation and process reengineering rather than in hard competition with other SMEs.

SMEs present positive qualities to behave like network nodes thanks to their lean structure, adaptability to market evolution, active involvement of versatile human resources, habit to establish sub-contracting relations, good technological level of their products. On the other side, the main obstacle to co-ordination is the traditional individualistic attitude of SMEs, that leaves each company completely alone in facing marketing, purchasing, design, engineering and technological innovation problems.

Besides legal and administrative aspects, the construction of a SME network requires the availability of proper software tools [4]. Their role is twofold: assure that the network reaction to customer request is as efficient as that of a single large enterprise, and warrant the nodes that the network planning policy is applied correctly and impartially. Formalising the SME network organisation, designing and developing the support software tools, validating the achieved results at a number of real cases, are the main objectives of the ESPRIT project 20723 - PLENT (PLanning small-medium Enterprise NeTworks). The project start date is January 1996 and its duration is 30 months.

Following the PLENT approach, the network is conceived as a set of operational nodes with equal rights, co-ordinated by a central unit that interacts with customers, distributes tasks to nodes on the basis of prefixed criteria and up-to-date information on node state and reliability, and informs each node on its position in the network with respect to the manufacturing process. The nodes are responsible of executing the assigned tasks within given time intervals and, to this purpose, they maintain direct relations with the respective suppliers and clients and try to solve possible network perturbations.

The PLENT project is primarily concerned with distributing and planning manufacturing activities. The focus on manufacturing arises from the fact that these networks are usually constituted for production purposes, hence they mainly require effective support to manufacturing co-ordination. Subsequently, nodes with specialised service functions will be included, thus extending types and number of activities to co-ordinate.

The role of the co-ordinating unit is fundamental for network operation [4]; its main functions are: (i) manage the operational schemes of the network products, expressed in terms of the macro-phases that can be carried out by the different nodes; (ii) manage the representation of work capacities of the different nodes, with respect to each macro-phase; (iii) manage the representation of network and node performances, to evaluate node qualification and reliability; (iv) manage customer orders, and decide their subdivision into balanced orders to the network nodes; (v) send orders to the respective nodes, informing them on their position in that specific productive chain; (vi) arrange new workload distributions in 
case of problems that cannot be damped down within the network; (vii) perform simulation activities to evaluate network reactions with respect to planned or foreseen market demands.

This calls for the design and development of two software modules, a co-ordination module and a performance evaluation module. It is the aim of this paper to analyse the most important requirements of the co-ordination module. Section 1 introduces representations of product operational schemes and of work capacities made available by nodes to the network (with respect to manufacturing macro-phases). Section 2 presents the basic co-ordination principles and the co-ordinating unit workload distribution rules derived from them. In Section 3, some simple cases are presented to show the effects of this policy in exemplary situations.

\section{NETWORK PROCESS REPRESENTATION}

In order to correctly distribute tasks to the network nodes, the co-ordinating unit must have a clear knowledge of the manufacturing process phases that each node can perform. This does not require that every transformation and assembly operation is modelled; rather, process phases are identified that correspond to the complex activities carried out by the nodes before they send materials to other nodes.

We call operational schema the direct acyclic graph of process phases and transportations that concur to realise a given product (Figure 1). Its structure is based on the following rules: (i) each transportation is characterised by the specific material transported and constitutes the link between the phases that produce and consume it; (ii) if two or more phases produce a given material, thus concluding alternative paths, they deliver it through a unique transportation, the same transportation supplying the material to the one or more phases consuming it; (iii) a final transportation is introduced to represent delivery of the finished product to the customer; (iv) each non-leaf phase receives materials from one or more transportations and delivers the resulting material to the corresponding transportation; (v) whenever a node produces different materials from the set of those received, as many distinct phases are identified; (vi) leaf phases are those including the earliest manufacturing activities, hence they do not receive materials from network nodes.

The only materials of interest for the co-ordinating unit are those resulting from process phases, that is, those involved in network transportations. All the other materials consumed during phase execution, including those feeding the leaf phases, come from outside the network: planning and organising their timely availability are tasks of the node performing the single phase.

In general, a network offers different versions of its product, that is, a family of products sharing most phases and transportations. For network co-ordination purposes, and to avoid redundancies, it is useful to introduce a family operational schema in the analogy with that defined in $[1,2,3]$. The family operational schema presents manufacturing alternatives: alternative transportations indicate the production of different component types for different product versions; alternative phases that lead to the same component type indicate execution of different processes that do not impact on the following phases. Once the customer has chosen the desired version, the corresponding operational schema is obtained by cutting the alternatives paths that are not of interest. 


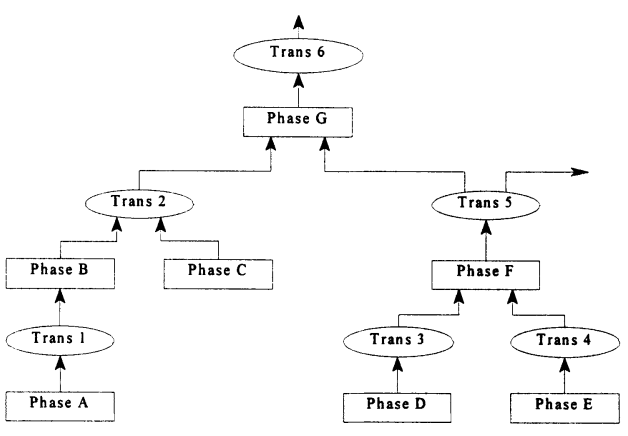

Figure 1: Fragment of network operational schema

Phase and transportation definition depends on the specializations of the network nodes. Each node is required to declare which activities it intends to execute with respect to the product process breakdown, and on this basis the network defines the operational schema. Whenever a new node reaches the network, or an existing node leaves it or changes its manufacturing capability, the operational schema is revised. The representation of phases and transportations is completed by durations and capacities.

Since the network nodes are geographically distributed, the transportation times from nodes producing a product to nodes consuming it can substantially differ. Hence, a time (typically expressed in days) is associated to every pair of nodes linked by that transportation. Effective task distribution among nodes must take into account this aspect; as we shall see, the task distribution algorithm is not affected by transportation capacity problems.

By declaring its own capacity for a given phase a node authorises the co-ordinating unit to charge it up to that limit, and ensures it will be able to meet the planned due dates. The node productive capacity for a given phase refers to three basic dates: the order date Do (the day when the order arrives from the co-ordinating unit), the phase start date Ds (the day when the materials to work are available and phase execution actually begins) and the first delivery date $\mathrm{Dd}$ (the day when the first batch is delivered by the node). We call notice time TN the minimum interval between Do and Ds accepted by the node; work latency time $T W$ the average interval between Ds and Dd; daily capacity $\boldsymbol{C P}$ the number of pieces worked every day from $\mathrm{Dd}$ on. The lower $T N$ and $T W$, the higher the possibility to have assigned tasks from the co-ordinating unit.

$T N$ is declared by the node on the basis of the time it spends to schedule the order for production and the time needed to have available the marginal components acquired from outside the network. A shorter $T N$ is obtained when purchasing components on forecast, without waiting for the communication of the single order. This is critical for the nodes performing leaf phases, since they have also to purchase the product base (rough) materials. In its turn, $\boldsymbol{T W}$ is declared by the node taking into account the phase global execution time, that is, the number of days needed in the average to transform the materials provided by the 
supplier nodes into the output material of that phase. $\boldsymbol{T W}$ is not easily shortened, the only way being process re-engineering towards a flow-shop organisation (if applicable).

A general representation of the node work capacity is given by the ratio between a number of pieces and a number of days. The following are examples of capacity for phases $A, B$ and C: $\boldsymbol{C P}(\mathrm{A})=50$ (50 pieces a day), $\boldsymbol{C P}(\mathrm{B})=100 / 3$ (100 pieces every 3 days), $\boldsymbol{C P}(\mathrm{C})=1 / 5(1$ piece every 5 days). Observe that the three capacities in the example can be declared by the same node, to inform the co-ordinating unit that it is able to carry out the phases A, B and C. Capacity declaration do not refer explicitly to the node manufacturing resources, which are only known to the local scheduling package [4]. However, capacity limits on concurrent phases can be placed by means of the "|" operator. For instance: $\boldsymbol{C P}(\mathrm{A})=200 \mid \boldsymbol{C P}(\mathrm{B})=160$ indicates that in a day the node can produce up to 200 pieces from Phase $A$ or 160 pieces from Phase B (or proper combinations of the two productions, as we shall see).

Finally, the node can declare a minimum batch size $\boldsymbol{M B}$ for a given phase, for instance: $\boldsymbol{M B}(\mathrm{A})=10$. It means that the co-ordinating unit is not authorised to charge the node for that phase with a batch whose size is lower than $\boldsymbol{M B}$. Declaring a $\boldsymbol{M B}$ value has two main consequences: first, the node is not considered when distributing small customer orders; second, every customer order that do not overcome the minimum batch size of one or more phases is rejected. The former effect damages the node that imposes such threshold, the latter damages the whole network. Then, minimum batch sizes must be strongly justified and negotiated within the network consortium.

\section{WORKLOAD DISTRIBUTION}

The co-ordinating unit must be in condition of accepting and confirming network orders without consulting with the involved nodes. This is done by splitting customer orders into phase orders, that are distributed to the nodes on the basis of the declared time constraints and productive capacities. For pursuing this general objective the co-ordinating unit applies a number of rules, the most important of which are examined in the following.

Rule 1: One order at a time. Splitting customer orders into node tasks is performed order by order. Since the external request should be answered in the shortest time, the co-ordinating unit cannot cumulate it with other orders for optimisation purposes, but must process it immediately. Optimisation is up to the single node, which remains completely responsible of its own planning policy.

Rule 2: Phase assignment at the latest. To ensure the widest time margins for order processing at the individual node, phases are assigned to nodes in a backward fashion, that is, going through the operational schema from root to leaves. The only constraints to the phase assignment algorithm are meeting the customer order due date and taking into account the tasks already distributed to nodes.

Rule 3: Assignment of daily workloads. For each phase, the workload assignment algorithm minimises the time, in days, occupied by phase execution. Starting from the phase due date, as it results from the backward analysis, the algorithm computes the network daily available capacity and verifies if it is sufficient to run out the batch size; if not, the exceeding quantity is transferred to the previous day and the allocation procedure is repeated. 
Rule 4: Balanced node involvement. The network nodes have equal rights, hence the assigned workloads must be proportional to the respective available capacities. The daily available capacity declared by a node is decreased to take into account previous assignments. If two or more nodes are able to perform that phase, each of them is charged for an amount proportional or equal to its available capacity, depending on whether the workload to distribute is less than the total available capacity or not. This rule pushes nodes to investments that can increase productivity.

Rule 5: Minimum impact of transportation times. The algorithm tries to keep transportation delays as close as possible to their minimum, which is obtained when the supplier daily production is immediately transferred to the client. This is done by considering each daily requirement as an independent order to be satisfied by the supplier nodes. The resulting phase overlapping gives the nodes further time margins to schedule their activities and does not prevent them from applying proper batch aggregation and production anticipation policies.

Rule 6: Lost days in workload distribution. The algorithm assigns daily workloads to nodes provided they are in condition to execute them, excluding a node from workload distribution when it is not productive, that is, during transportation, work latency and notice times. In such days, the concurrent nodes get all the workload. Since fast-reacting nodes are favoured, this rule pushes towards leaner and more efficient organisations.

Rule 7: Node internal concurrence. Workload distribution must take into account concurrent capacity declarations. To this purpose, whenever a task is charged onto a node the remaining capacity is decreased for both the corresponding phase and each of its concurrent phases.

Rule 8: Effect of node reliability. The network performance evaluation module gives a reliability measure of each node with respect to each performed phase, in the form of a percentage that becomes less than $100 \%$ when node reliability decreases, and more that $100 \%$ when the node gains consideration, e.g. for damping down perturbations. In the former case the reliability percentage can be used to reduce proportionally the node declared capacity; in the latter case it may represent a title to further capacity claims.

It may happen that for a given order no tasks distribution can be found that satisfies the above constraints. This means that node capacities, decreased by previous assignments, are not sufficient to deliver the required amount of products within the due date. In this situation either due date and batch size are re-negotiated with the customer or network nodes have to declare higher capacities, at least for the period of time covered by the order.

\section{SIMPLE EXAMPLES}

To exemplify the effects of applying the above rules, we consider in this section some simple cases of workload distribution, referring to the phases and transportations reported in Figure 1. In the following, workload assignments are represented by tables, a cell per day; the cell content is numerical or literal: the former adopts the format WL:CP, where WL stands for assigned workload and CP for capacity; the latter takes the value $\mathrm{W}$ to indicate a work latency day and the value $\mathrm{N}$ to indicate a notice (planning) day.

Let us start with Phase G. The first hypothesis is that only Node $\alpha$ performs it, with $\boldsymbol{C P} \alpha(\mathrm{G})=30$ and $\boldsymbol{T W} \alpha(\mathrm{G})=2$. Assuming an order size of 220 pieces, and no previous workload allocation, the charge distribution results: 


\begin{tabular}{cccccccccccc|c}
\hline 12 & 11 & 10 & 9 & 8 & 7 & 6 & 5 & 4 & 3 & 2 & 1 & Days to due-date \\
\hline $0: 30$ & $0: 30$ & W & W & $10: 30$ & $30: 30$ & $30: 30$ & $30: 30$ & $30: 30$ & $30: 30$ & $30: 30$ & $30: 30$ & Node $\alpha$ \\
\hline
\end{tabular}

If also Node $\beta$ can perform Phase $G$, with $\boldsymbol{C P} \beta(\mathrm{G})=45 / 2$ and $\boldsymbol{T} \boldsymbol{W} \beta(\mathrm{G})=2$, the following new distribution is obtained:

\begin{tabular}{cccccccccccc|c}
\hline 12 & 11 & 10 & 9 & 8 & 7 & 6 & 5 & 4 & 3 & 2 & 1 & Days to due-date \\
\hline $0: 30$ & $0: 30$ & $0: 30$ & $0: 30$ & $0: 30$ & $\mathrm{~W}$ & $\mathrm{~W}$ & $4: 30$ & $30: 30$ & $30: 30$ & $30: 30$ & $30: 30$ & Node $\alpha$ \\
$0: 45 / 2$ & $0: 45 / 2$ & $0: 45 / 2$ & $0: 45 / 2$ & $\mathrm{~W}$ & $\mathrm{~W}$ & $0: 0$ & $6: 45$ & $0: 0$ & $45: 45$ & $0: 0$ & $45: 45$ & Node $\beta$ \\
\hline
\end{tabular}

The next case examines the pair constituted by Phase $G$ and Phase $F$, the latter being performed by the only Node $\gamma$, with $\boldsymbol{C P} \gamma(\mathrm{F})=50$. Transportation times are: $\boldsymbol{T} \boldsymbol{T} \gamma \alpha=1, \boldsymbol{T} \boldsymbol{T} \gamma \boldsymbol{\beta}=2$. The table shows a tentative workload distribution to Node $\gamma$ that minimises the impact of transportation but neglects the daily capacity limit.

\begin{tabular}{cccccccccccc|c}
\hline 12 & 11 & 10 & 9 & 8 & 7 & 6 & 5 & 4 & 3 & 2 & 1 & Days to due-date \\
\hline $0: 30$ & $0: 30$ & $0: 30$ & $0: 30$ & $0: 30$ & $\mathrm{~W}$ & $\mathrm{~W}$ & $4: 30$ & $30: 30$ & $30: 30$ & $30: 30$ & $30: 30$ & Node $\alpha$ \\
$0: 45 / 2$ & $0: 45 / 2$ & $0: 45 / 2$ & $0: 45 / 2$ & $\mathrm{~W}$ & $\mathrm{~W}$ & $0: 0$ & $6: 45$ & $0: 0$ & $45: 45$ & $0: 0$ & $45: 45$ & Node $\beta$ \\
& & & $4: 50$ & $30: 50$ & $30: 50$ & $30: 50$ & $30: 50$ & & & & & Node $\gamma$ to $\alpha$ \\
& $6: 50$ & & $45: 50$ & & $45: 50$ & & & & & & & Node $\gamma$ to $\beta$ \\
\hline
\end{tabular}

In order to achieve a correct distribution, the exceeding workloads must be anticipated. The following tables report the final results obtained by anticipating deliveries to Node $\alpha$ or to Node $\beta$, respectively. Note that the planned activities for Node $\gamma$ coincide in the two cases.

\begin{tabular}{|c|c|c|c|c|c|c|c|c|c|c|c|c|}
\hline 12 & 11 & 10 & 9 & 8 & 7 & 6 & 5 & 4 & 3 & 2 & $I$ & Days to due-date \\
\hline $0: 30$ & $0: 30$ & $0: 30$ & $0: 30$ & $0: 30$ & $\overline{\mathrm{W}}$ & $\overline{\mathrm{W}}$ & $4: 30$ & $30: 30$ & $30: 30$ & $30: 30$ & $30: 30$ & Node $\alpha$ \\
\hline \multirow[t]{3}{*}{$0: 45 / 2$} & $0: 45 / 2$ & $0: 45 / 2$ & $0: 45 / 2$ & W & W & $0: 0$ & $6: 45$ & $0: 0$ & $45: 45$ & $0: 0$ & $45: 45$ & Node $\beta$ \\
\hline & & $4: 50$ & $5: 50$ & $50: 50$ & $5: 50$ & $30: 50$ & $30: 50$ & & & & & Node $\gamma$ to $\alpha$ \\
\hline & $6: 50$ & & 45:50 & & 45:50 & & & & & & & Node $\gamma$ to $\beta$ \\
\hline 12 & 11 & 10 & 9 & 8 & 7 & 6 & 5 & 4 & 3 & 2 & 1 & Days to due-date \\
\hline $0: 30$ & $0: 30$ & $0: 30$ & $0: 30$ & $0: 30$ & $\overline{\mathrm{W}}$ & $\overline{\mathrm{W}}$ & $4: 30$ & $30: 30$ & $30: 30$ & $30: 30$ & $30: 30$ & Node $\alpha$ \\
\hline \multirow[t]{3}{*}{$0: 45 / 2$} & $0: 45 / 2$ & $0: 45 / 2$ & $0: 45 / 2$ & W & W & $0: 0$ & $6: 45$ & $0: 0$ & $45: 45$ & $0: 0$ & $45: 45$ & Node $\beta$ \\
\hline & & & $4: 50$ & $30: 50$ & $30: 50$ & $30: 50$ & $30: 50$ & & & & & Node $\gamma$ to $\alpha$ \\
\hline & $6: 50$ & $4: 50$ & $46: 50$ & $20: 50$ & $20: 50$ & & & & & & & Node $\gamma$ to $\beta$ \\
\hline
\end{tabular}

Finally, consider a leaf phase, like Phase A, performed concurrently by Node $\delta$ and Node $\varepsilon$, with $\boldsymbol{C P} \delta(\mathrm{A})=15, \boldsymbol{C P} \varepsilon(\mathrm{A})=35, \boldsymbol{T} \boldsymbol{W} \delta(\mathrm{A})=1, \quad \boldsymbol{T} \boldsymbol{W} \varepsilon(\mathrm{A})=3, \boldsymbol{T N} \delta(\mathrm{A})=3$ and $T N \varepsilon(\mathrm{A})=5$. The first tables presents a distribution not affected by notice time, in that the 12th day is hypothesised far from the current date. The second table presents the distribution that results assuming the 12th day corresponding to the current date. In this case, some days are lost for workload assignment, according to Rule 6; Node $\delta$ takes advantage of its shorter notice and latency time while, in the former case, Node $\varepsilon$ took advantage of its higher daily capacity.

\begin{tabular}{cccccccccccc|c}
\hline 12 & 11 & 10 & 9 & 8 & 7 & 6 & 5 & 4 & 3 & 2 & 1 & Days to due-date \\
\hline $0: 15$ & $0: 15$ & $0: 15$ & $0: 15$ & $0: 15$ & $0: 15$ & $\mathrm{~W}$ & $6: 15$ & $15: 15$ & $15: 15$ & $15: 15$ & $15: 15$ & Node $\delta$ \\
$0: 35$ & $0: 35$ & $0: 35$ & $0: 35$ & $\mathrm{~W}$ & $\mathrm{~W}$ & $\mathrm{~W}$ & $14: 35$ & $35: 35$ & $35: 35$ & $35: 35$ & $35: 35$ & Node $\varepsilon$ \\
\hline
\end{tabular}




\begin{tabular}{cccccccccccc|c}
\hline 12 & 11 & 10 & 9 & 8 & 7 & 6 & 5 & 4 & 3 & 2 & 1 & Days to due-date \\
\hline $\mathrm{N}$ & $\mathrm{N}$ & $\mathrm{N}$ & $0: 15$ & $0: 15$ & $\mathrm{~W}$ & $5: 15$ & $15: 15$ & $15: 15$ & $15: 15$ & $15: 15$ & $15: 15$ & Node $\delta$ \\
$\mathrm{N}$ & $\mathrm{N}$ & $\mathrm{N}$ & $\mathrm{N}$ & $\mathrm{N}$ & $\mathrm{W}$ & $\mathrm{W}$ & $\mathrm{W}$ & $35: 35$ & $35: 35$ & $35: 35$ & $35: 35$ & Node $\varepsilon$ \\
\hline
\end{tabular}

\section{CONCLUSIONS}

With this paper we have analysed and discussed the basic principles that inspire the coordination policy of SME networks. In particular, we have shown how the objective of assigning balanced workloads to network nodes can be achieved by the application of proper rules. These rules constitute the basis of one of the three software prototypes that will result from the cited PLENT project. In order to ensure that the PLENT approach corresponds to the actual needs of different SME networks, the project foresees the execution of the following steps: (i) extensive requirement collection at existing and forthcoming networks; (ii) planning policy formalization; (iii) planning policy validation and refinement; (iv) software design and development; (v) software validation at the existing networks; (vi) organisation of new networks according to the PLENT approach. For the sake of completeness, it should be recalled that the countries involved in this project are Italy, Greece, Spain and Hungary.

\section{REFERENCES}

[1] Bonfatti F., Monari P. D., Paganelli P.: "Towards a rule-based unified product modelling", DKSME 94 (Database and Knowledge Systems for Manufacturing and Engineering) Int'l Conf., Hong Kong, 1994.

[2] Bonfatti F., Monari P. D., Paganelli P.: "Modelling process by rules: regularities, alternatives and constraints", IMSE '94 (Integrated Manufacturing Systems Engineering) European Conf., Grenoble, France, 1994.

[3] Bonfatti F., Monari P. D., Paganelli P., "Modelling manufacturing resources and activities: an ontology", ICCIM '95 (Computer Integrated Manufacturing) Int'l Conf., Singaporz, 1995.

[4] Bonfatti F., Monari P. D.: "Planning small-medium enterprise networks", IMPDE 95 (Improving Manufacturing Performance in a Distributed Enterprise) Int'l Conf., Edinburgh, 1995.

[5] Hirsch B. E., Crom S., "Management challenges in globally distributed production environments", IFIP WG 5.7 Working Conf. on Evaluation of Production Management, Gramado, Brazil, 1994.

[6] Hirsch B. E. et al., "Decentralized and collaborative production management in distributed manufacturing environments", in J. H. K. Knudsen et al. (eds), Sharing CIME Solutions, Ios Press, 1995.

[7] Hopf M., Holonic manufacturing systems (HMS) - the basic concept and a report of IMS Test Case 5, in J. H. K. Knudsen et al. (eds), Sharing CIME Solutions, Ios Press, 1994.

[8] Rolstadas A., "Beyond Year 2000 - Production Management in the Virtual Company", IFIP Transactions B-19: Production Management Methods, North Holland 1994. 